\title{
ENTRE GAMERS E HACKERS: REFLEXÕES SOBRE A CULTURA DIGITAL
}

\author{
BETWEEN GAMERS AND HACKERS: THOUGHTS ON THE DIGITAL CULTURE \\ ENTRE JUGADORES Y HACKERS: REFLEXIONES SOBRE LA CULTURA DIGITAL \\ José Messias ${ }^{1}$ \\ Letícia Perani²
}

Resumo: o presente artigo apresenta uma aproximação entre o jogo e a cultura hacker, realizada a partir de uma abordagem histórica e filosófica. Ambos serão investigados pelos seus potenciais de ligação com as relações entre os humanos e as tecnologias, sobretudo o seu papel na difusão e atual estado da cultura digital. Além da trajetória compartilhada, as condições de existência dessas figuras estão entrelaçadas pelos vínculos afetivos e pelas propriedades de seus suportes materiais. Assim, constituído em formato de ensaio, estão destacados o convite e a ação, e a configuração e a customização como partes integrais da experiência de jogo e motores da cultura hacker, e principalmente os potenciais dessas ações ao saírem do âmbito virtual e incluírem ações antes mesmo do ato de jogar. Este percurso iniciado com uma breve discussão sobre os modos de letramento e as materialidades acionadas por esses objetos técnicos digitais estabelece o lúdico como ponto de convergência dessas práticas.

Palavras-chave: Cultura hacker. Games. Lúdico.

Abstract: this paper presents the possible similarities between gaming and the hacker culture. In this historical and philosophical approach, both are considered in their po-

- Doutor em Comunicação e Cultura pela Escola de Comunicação da Universidade Federal do Rio de Janeiro (UFRJ). Pós-Doutorando (Capes/PNPD) no Programa de Pós-Graduação em Comunicação da Universidade Federal Fluminense (UFF), Niterói, RJ, Brasil. http://orcid.org/o000-0001-6950-220X. E-mail: jmessias.santos@gmail.com

2 Doutora em Comunicação pelo Programa de Pós-Graduação em Comunicação da Universidade do Estado do Rio de Janeiro (UERJ). Professora Adjunta do Instituto de Artes e Design da Universidade Federal de Juiz de Fora - UFJF, Juiz de Fora, MG, Brasil. http://orcid.org/oooo-0002-9916-996X. E-mail: leticiaperani@yahoo.com.br 
tential to establish connections between human and technology, mainly their role in the expansion and current state of digital culture. Beyond their shared path, the modes of existence of these figures are intertwined by affective attachments and the attributes of their material grounding. We highlight the invitation to action, configuration and customization as inherent parts of the gaming experience and driving forces of hacker culture. Most importantly, these actions are not limited to in-game or computational activities, they expand to a set of decisions and proceedings performed even before playing starts. Starting from a brief discussion on the modes of literacy and materialities evoked by theses digital technical objects, the ludic emerges as point of convergence between theses practices.

Keywords: Hacker culture. Games. Ludic.

Resumen: este artículo presenta las posibles similitudes entre el juego y la cultura hacker. En este enfoque histórico y filosófico, ambos se consideran en su potencial para establecer conexiones entre el ser humano y la tecnología, principalmente su papel en la expansión y el estado actual de la cultura digital. Más allá de su camino compartido, los modos de existencia de estas figuras están entrelazados por los afectos y los atributos de su fundamento material. Destacamos la invitación a la acción y la configuración / personalización como partes inherentes de la experiencia de juego y las fuerzas motrices de la cultura hacker. Lo que es más importante, estas acciones no se limitan a actividades de juego o computacionales, sino que se amplían a un conjunto de decisiones y procedimientos realizados incluso antes de que comience el juego. A partir de una breve discusión sobre los modos de alfabetización y materialidades evocados por estos objetos técnicos digitales, el lúdico surge como punto de convergencia entre estas prácticas.

Palabras clave: Cultura hacker. Games. Lúdico.

\section{Introdução}

A experiência de jogo é tema de interesse para o campo dos Game Studies, mas também para as áreas da Cibercultura ou das Humanidades Digitais de maneira geral. A inegável presença dos jogos digitais na sociedade contemporânea, o impacto econômico de sua indústria nas cifras das dezenas de bilhões de dólares, assim como a importância cultural do jogo/lúdico e seus efeitos cognitivos (não apenas em sua vertente digital), evidenciam a relevância do tema. Neste contexto, este texto procura seguir uma linha de investigação genealógica (DELEUZE, 
2018) e epistemológica, relacionando essa experiência com outro elemento de destaque dentro do meio, a cultura hacker.

O ponto de encontro suscitado por este artigo é o ato, ou melhor, um impulso de modificação e de ações não triviais que residiria em ambos. As ações realizadas antes do jogo para customizar (e muitas vezes piratear) seriam análogas a esse chamado para tornar ou retomar esse aspecto livre da tecnologia - liberdade de acesso, controle e manipulação - de que, em última análise, se trata a cultura hacker (WARK, 2004; GALLOWAY, 2004). Nesse âmbito, política e entretenimento (digital) se encontram como vetores dos agenciamentos entre hackers e gamers.

Essa relação será trabalhada em diversos vieses, em primeiro lugar, no material no qual o contato com computadores e aparatos derivados promove uma espécie de letramento/alfabetização tecnológica e midiática que capacita indivíduos e ajudou a estabelecer o que se entende por culturas hacker e gamer. Nos âmbitos epistemológico e genealógico, confrontou-se diferentes visões sobre as teorias do lúdico e sobre a experiência de jogo em busca destas propriedades, espelhando-as, quando necessário, com a história da computação e do design. Deste modo, busca-se encontrar alguns dos princípios e condições comuns de existência de ambos.

\section{Letramento digital na cultura hacker e nos games}

Em sua pesquisa sobre fãs de música, que ele chama de amantes/amadores ${ }^{3}$ (amateurs), o sociólogo francês Antoine Hennion ressalta o papel dos "vínculos ou vinculações ${ }^{\prime \prime}$ (attachments) em detrimento até da própria noção de gosto. Para ele, o fã ou amador está envolvido num arranjo sociotécnico, e as mediações que ocorrem em seu interior muitas vezes independem da vontade desses amadores. $\mathrm{O}$ vínculo mostra como eles determinam e são determinados pelos objetos de suas afecções, e ainda como os comportamentos que surgem e os aparatos mobilizados são todos frutos dessa ligação. Segundo ele:

Essa bela palavra [vinculação] destrói a oposição que acentua o dualismo da palavra "gosto", entre uma série que vem de fora e o "hic et nunc" [aqui e agora] da situação e da interação. No que se refere aos fãs de música, há menos uma ênfase em rótulos e mais em estados,

\footnotetext{
3 Hennion (2010) propositalmente brinca com significado da palavra amateur em francês e inglês, chamando a atenção para raiz/origem na palavra amor.

4 Ligação ou pertencimento talvez fossem traduções mais literais de attachment, contudo, optou-se por seguir a tradução proposta por Amaral e Monteiro (2013).
} 
menos em autodeclaração e mais nas atividades das pessoas; a respeito dos objetos que motivam esse gosto, seu direito de resposta e sua habilidade de coproduzir o que está acontecendo, o que surge do contato permanece aberto5 (HENNION, 2010, p. 26 - grifos e tradução nossos).

Esses vínculos, mais do que objetos específicos, opiniões, crítica especializada e até conhecimento sobre o tema, condicionam a experiência afetiva musical (e cultural de maneira geral), tornando possível as associações entre os elementos individuais. Esta performance situada, material e temporalmente construída, dentro da chamada cultura participativa possui relação com uma noção contemporânea e constantemente negociada de hacker e seu duplo frequentemente omitido, o pirata (WARK, 2004). Nesse contexto, defende-se que o mesmo argumento feito por Hennion sobre a natureza dessas vinculações pode ser utilizado com os games.

Computadores e videogames, objetos trabalhados neste estudo são, a grosso modo, são ambientes midiáticos interativos dotados de capacidades multimídia e multitarefa. Além disso, não há como negar a existência de uma demanda por certa proficiência com computadores na sociedade dita pós-industrial ou pós-fordista. Castells (2003), Negri e Hardt (2005, 2010), Lazzarato (2006), entre tantos outros, identificam que a ascensão das Tecnologias de Informação e Comunicação (TIC) coloca os computadores no epicentro de um movimento global. Aquilo que se convencionou chamar de sociedade da informação (e outros nomes derivados). Entretanto, menos os computadores como aparatos tangíveis, mas a absorção à lógica de produção do processamento de dados, a circulação de informação em velocidades e quantidades jamais vistas e a convergência de formatos e linguagens para o digital se tornam o foco dessa apropriação.

Assim, surge efetivamente uma cultura digital que deixa de ser um nicho concentrado em laboratórios da Big Science norte-americana - universidades e multinacionais - para ser introduzida paulatinamente na vida da população. Com ela também nasce todo um campo de estudos dentro das ciências cognitivas e áreas correlatas, que fala do aprendizado e da incorporação dessas tecnologias, possibilitando assim, falar de uma alfabetização computacional, ou mais precisa-

\footnotetext{
5 Do original: This beautiful word [attachment] destroys the opposition that accentuates the dualism of the word «taste», between a series of causes that come from outside and the «hic et nunc» of the situation and the interaction. In terms of music fans, there is less emphasis on labels and more on states, less on self-proclamations and more on people's activity; regarding the objects that motivate taste, their right to reply and their ability to coproduce what is happening, what arises from the contact, remains open.
} 
mente, letramento midiático e digital ${ }^{6}$ (media and digital literacy). Nesse sentido, para os fins da presente argumentação, o que os computadores proporcionam é mais importante do que o que eles são. Os dois não estão separados, mas neste estudo a segunda está a serviço da primeira. Ou seja, o letramento também como "produto" cognitivo desses vínculos.

Como mostram pesquisas de Mäyrä (2008), Natale (2013), Levy (2010), entre outros, historicamente os videogames têm um papel central no desenvolvimento e popularização dos computadores. Não só computação e videogames, mas também a cultura hacker estão intrinsecamente ligados desde o início. Eles fazem parte de um arranjo sociotécnico que coloca em relação afetos, materialidades e competências cognitivas. No entanto, acredita-se que esse arranjo não seja estanque, adquirindo diferentes configurações no decorrer do tempo e dependendo do contexto.

O caso de Steve Russell e a criação do game Spacewar! (Guerra no Espaço) ${ }^{7}$ como forma de testar a potencialidade do minicomputador PDP-1, em 1962, é emblemático para uma breve rememoração histórica. Isso porque Russell e seus colegas do Instituto de Tecnologia de Massachusetts (MIT) chamavam o projeto de "display hack" (hack ou gambiarra usando telas ${ }^{8}$ ), o que evidencia esse imbricamento entre jogos eletrônicos e a cultura hacker. Levy (2010) conta que Russel e companhia estavam interessados em criar uma aplicação para o equipamento recém-chegado no laboratório, uma vez que era comum nesse primeiro estágio da computação os próprios usuários desenvolverem os programas que seriam adicionados ao sistema operacional ou até mesmo o próprio (caso julgassem necessário). Este era um dos principais atrativos dos computadores na época, e um dos motivos de sua popularização: estar associado a uma comunidade de desenvolvedores - pessoas com formação ou algum treinamento prévio (mesmo que informal) em programação/informática. Da mesma forma, o físico William Higinbotham, desejando atrair a atenção dos visitantes do laboratório nuclear

\footnotetext{
6 Letramento pode ter significados diferentes. Pode ser um sinônimo de alfabetização, no sentido de aprender "as letras". Ou, um sentido mais amplo, seria a aquisição de competências relacionadas às práticas comunicativas diversas (multimodais). Há ainda uma corrente nos estudos de comunicação e design no Brasil, que prefere traduzir literacy diretamente para literacia, para evitar essa ambiguidade com um conceito exclusivamente aplicado à escrita. Para este trabalho, adotaremos o termo mais corrente, letramento.

7 Spacewar! (MIT,1962)

8 Uma aproximação entre a palavra hack, em inglês, que pode ser verbo (to hack) e substantivo e o termo gambiarra não é novidade, e pode até ser encontrada na página da Wikipédia do verbete hacker. WIKIPEDIA (Org.). Hacker. 2015. Disponível em: https://pt.wikipedia.org/wiki/Hacker. Acesso em: 24 ago. 2015.
} 
onde trabalhava, "hackeou" um osciloscópio para criar um dos primeiros videogames ${ }^{9}$, em 1958.

Esses dois casos da pré-história da cultura gamer ilustram bem algumas das atribuições e propriedades dos suportes tecnológicos de um modo de existência dos games e do hacker. Esses casos remetem à discussão proposta por Gilbert Simondon (2017) originalmente também em 1958. O autor desloca o conhecimento produzido até então sobre objetos técnicos, de matriz substancialista, para uma ideia que privilegia o processo: estabilidade momentânea ao invés de repouso, multiplicidade de potenciais ao invés de unidade. A configuração visível (e inteligível) do indivíduo sendo apenas um estágio - um dos estágios finais, mesmo que não o último - da realização desse processo, do processo de individuação. Segundo ele:

[...] podemos afirmar que a individuação de entidades técnicas é a condição essencial para o progresso técnico. Esta individuação é possível por causa da recorrência de causalidade no ambiente que a entidade técnica cria em torno de si, um ambiente que ela influencia e pelo qual também é influenciada. Esse ambiente, ao mesmo tempo natural e técnico, pode ser chamado de meio associado ${ }^{10}$ [associated milieu] (SIMONDON, 2017, p. 59, tradução nossa).

O mesmo vale para a sociologia das associações de Bruno Latour (2005, 2013). Essas associações "ditam" como os construtos e fenômenos antes chamados de sociais vão se organizar, formando assim, o que entendemos por sociedade. Para Latour, falar em modo de existência da tecnologia seria diferente de falar em um modo de existência de um objeto técnico, um termo que remete a uma unidade ou identidade, o que privilegiaria uma noção reificada, ao invés de uma qualidade. De acordo com o autor, "'tecnologia' não designa um objeto, mas uma diferença, uma exploração inteiramente nova do ser-como-outro, uma nova declinação da alteridade"11" (LATOUR, 2013, p. 223, tradução nossa).

\footnotetext{
9 Natale (2013) explica que um osciloscópio é um aparelho para aferição de voltagem que consiste basicamente numa tela e em fio condutor sensível a cargas elétricas (catodo). Através das affordances rudimentares da tela do osciloscópio - similares a um televisor - o cientista conseguiu criar um jogo de tênis de mesa.

10 Do original: We can therefore affirm that the individualization of technical beings is the condition of technical progress. This individualization is made possible by the recurrence of causality within a milieu that the technical object creates around itself and that conditions it, just as it is conditioned by it. This simultaneously technical and natural milieu can be called an associated milieu.

1 Do original: 'technology' does not designate an object but rather a difference, an entirely new exploration of being-as-other, a new declension of alterity.
} 
Todavia, este estudo discorda da colocação do sociólogo francês quando ele afirma que esta preocupação não se faz presente na obra de Simondon (Latour, 2013). Em sua argumentação sobre o princípio da tecnicalidade, Simondon ressalta que o próprio humano costumava ser uma entidade técnica, dotado de tecnicidade, mas que precisou encontrar uma nova função dentro do conjunto/ agenciamento técnico (SIMONDON, 2017, p. 81). Ele ainda complementa defendendo que o objeto técnico é apenas uma figuração "momentânea" cuja função é justamente "transmitir" a tecnicidade (SIMONDON, 2017, p. 76). Ou seja, ao invés de se focar num aparato como um console específico ou um modelo de computador ou interface gráfica, o importante é estudar esses atributos, que são passados adiante (e algumas vezes rejeitados) nessa relação entre entidades e ambientes, e que estimulam ou produzem esses agenciamentos: o processo de individuação. Simondon sintetiza:

Tecnicidades podem ser concebidas como comportamentos estáveis refletindo as características dos elementos ao invés de simples qualidades. Elas são forças no sentido mais potente da palavra, o que significa que elas são capacidades de produzir ou sofrer um efeito de uma maneira determinada'2 (SIMONDON, 2017, p. 75, tradução nossa).

De fato, há uma forte confluência entre os computadores e os videogames, embora eles, de forma alguma, sejam considerados o mesmo objeto. No entanto, por ser uma mídia nativa do digital, segundo Mateas (2008), os últimos são uma importante porta para o que ele chamou de letramento processual (procedural literacy). Ele consiste basicamente na assimilação do modo de funcionamento do computador, sua estrutura lógica de funcionamento, que é a mesma dos videogames. Assim, a escalada do letramento processual, associada a uma atitude configurativamente lúdica, nos autorizaria a afirmar que um determinado jogador procurará por uma experiência customizada sempre que possível, mesmo que não lhe seja permitido. E essa lógica tem potencial para transcender o chamado mundo virtual.

Atualmente, há uma mudança em curso nas formas de assistir à televisão ou ler um livro. Práticas como comentário nas redes sociais, a produção de fanfics, modding, compartilhamento de montagens e memes etc., têm se tornado cada

12 Do original: Technicities can be thought of as stable behaviors, expressing the characteristics of elements, rather than as simple qualities: they are powers, in the fullest sense of the term, which is to say capacities for producing or undergoing an effect in a determinate manner. 
vez mais populares entre os consumidores de entretenimento. Ainda assim, há um grau de dificuldade ou esforço considerável em várias dessas ações, sobretudo no que se refere aos games (mas não somente a eles), que pode envolver edição de áudio, vídeo e som, produção de textos, como críticas e criação de grupo de discussão (GEE, 2015).

Por isso, ao customizar, esses jogadores estariam realizando uma ação análoga ao jogar, afirmação também presente na proposta de gaming literacy (letramento do jogar) de Eric Zimmerman (2009). Esse jogar com as estruturas, com as interfaces, ou mesmo com a proposta geral da interação com as mídias, dentro do paradigma digital e fora dele, pode ser entendido como jogar. A partir de Zimmerman, é possível afirmar que customizar e outras formas de intervenção não contradizem ou quebram as regras do jogo, fazem parte dele. O jogador joga o objeto, mas também as estruturas que o sustentam, o meio que o suporta, a comunidade ao redor dele. $\mathrm{E}$, nessa visão, isso configura um processo de conhecimento, um conhecer mediado pelo jogo/lúdico, que introjeta a configuração para além das barreiras materiais.

Com possibilidades de letramento midiático que contemplam a configuração como principal característica, os videogames parecem o modelo ideal para uma proposta que expanda essa lógica para abarcar as práticas de customização e personalização da cultura hacker. Não se trata de inventar uma nova mídia, linguagem ou ainda estabelecer uma ordem de importância de uma sobre outra (da "nova" sobre a "velha"), mas apenas apresentar uma perspectiva que trate da forma como os videogames estariam não só remediando outras mídias, como também nossa relação com elas. Segundo Gee e Haynes:

Jogadores usam os jogos como um centro de atividades que vão além de apenas jogar. Eles fazem mods; leem e escrevem sobre os jogos e sobre os temas envolvidos neles; e colaboram, geralmente pela Internet, não apenas para jogar juntos, mas pra criar mods, discutir o jogo e organizar todo o tipo de interação e aprendizado ao redor dos jogos e do jogari3 (2010, p. 38, tradução nossa).

A presença massiva dos games indicaria um paradigma tecnológico (material), que evocaria esse letramento não imersivo e não interpretativo defendido por

13 Do original: Gamers use games as a center for activities that go beyond just playing the game. They mod the games they play; they read and write about the games they play and about the themes these game involve; and they collaborate, often through the Internet, not just to play together, but to mod, to discuss the game, and to organize all sorts of interaction and learning around the game and gaming more generally. 
Moulthrop (2004), Malaby (2007), Consalvo (2009), entre outros, que é, ao mesmo tempo, afetivo e social (intangível). Esse paradigma é representado tanto pelas ações piratas como por formas menos "subversivas": como a introdução de jogos eletrônicos em sala de aula como complemento ao ensino, o treinamento de médicos em games e/ou simuladores, ou os games que dependem de conexão com redes sociais, extração de metadados e outros tipos de identificação, seja para conteúdos extras ou embutidos na mecânica do jogo.

Nesse sentido, a customização através dos mods ou outras formas menos invasivas apenas continua uma tendência da cultura digital (o conjunto de práticas, costumes e identidades surgido da inserção do computador na vida cotidiana) de participação do usuário que existia desde seus primeiros anos ainda na Big Science. Nesse período, a computação era relegada aos profissionais e acadêmicos de comprovada expertise técnica, com educação formal nas ciências exatas. Mesmo os curiosos e autodidatas, os amadores das primeiras eras digitais, que não contavam com uma educação formal, mas, possuíam algum tipo de treinamento ou capacitação técnica (sabiam programar, por exemplo). Todavia, na contemporaneidade constatou-se a existência de um aprendizado por demanda, baseado em uma forte cultura de tutoriais que independe de conhecimento prévio. Ela age por meio das interfaces gráficas e outros dispositivos lúdicos que utilizam diversas linguagens, que não são só verbais (Cf. REGIS, 2014; TIMPONI; REGIS, 2017). Esses jogadores-hackers podem não aprender a programar, mas adquirem habilidades que, mesmo transitórias, são o produto de um entrelaçamento cognitivo, só que menos galgado nas faculdades superiores da mente. Eles encontram formas intuitivas de se capacitar para realizar as modificações que desejam.

Videogames existem desde a segunda metade do século XX, mas seu status atual só foi atingido através do surgimento de toda uma cultura que os cerca, um aporte material, mas também social e cognitivo. Os mods são exemplos dessa busca por acesso e controle, impulsionada pelos games como mídia digital. A popularização e provável ubiquidade dos games como mídia na atualidade, saindo de uma tendência minoritária ou nicho geek, não representa apenas uma mudança de padrões de consumo.

Os games não andam sozinhos, eles são acompanhados de todo um ambiente, ou meio associado, como diria Gilbert Simondon (2017), que vai possibilitar e tornar visível e/ou identificável suas condições de existência. Uma delas seria a lógica ou propriedade configurativa (MOULTHROP, 2004; GALLOWAY, 2006, entre outros). Para os objetivos deste texto, a lógica ou qualidade configurativa 
representa a materialidade intangível mencionada anteriormente e, uma vez que ela está sendo difundida junto da popularização dos games, ela vai exercer sua agência também nesses indivíduos. $E$ isso marca justamente a passagem do reino da cultura tecnomeritocrática da ética hacker ressaltada por Castells (2003) e Silveira (2009), para as comunidades voltadas para o entretenimento. Ou seja, de uma comprovada expertise técnica ou intelectual para uma capacitação por demanda, situada e corporificada, mas por isso transitória, inconstante e até precária.

Por esse motivo, o hackerismo, pensado dentro de Castells (2003) como diferentes tipos de programação criativa, se mostra ideal para o prosseguimento desta discussão, pois remete, em última instância, a processos, formas criativas de uso do potencial do computador e da tecnologia digital. $\mathrm{O}$ autor, contudo, não ignora a ética hacker já estabelecida, que trata de uma busca por participação e igualdade, e, sobretudo, de um ideal de liberdade. Por isso, ele afirma, "suprema nesse conjunto de valores é a liberdade. Liberdade para criar, liberdade para apropriar todo conhecimento disponível e liberdade para redistribuir esse conhecimento sob qualquer forma ou por qualquer canal escolhido pelo hacker" (CASTELLS, 2003, p. 42).

A pirataria, por outro lado, estaria tradicionalmente relacionada a produtos, ou melhor, a (livre) apropriação de um determinado produto, bem ou técnica já estabelecida. Ela carrega implicitamente uma ideia de transgressão ou subversão, uma ruptura com a ideia de propriedade, seja uma patente, copyright ou posse de qualquer tipo. Embora não a utilizemos em um nível estritamente jurídico, o qual não nos interessaria por acreditarmos que possa restringir as possibilidades de aplicação do conceito, é importante ressaltar que não é porque ela necessariamente lida com algo existente que a pirataria não possa ser considerada uma ação criadora em si. Atos piratas operam rearranjos e recombinações e nesse processo reimaginam funcionalidades, aplicações e usabilidades, de forma a fazer emergir o novo. Em termos de práticas e estratégias, é possível ver que piratas e hackers se confundem (WARK, 2004) e, em nossa visão, apontam para diferentes formas de apropriação e/ou criatividade.

De um lado, na ética hacker tradicional, há uma tentativa de tornar a tecnologia digital mais justa e devolvê-la, dentro do sistema, a seu status inicial das décadas de 1960 e 1970, ou então construir uma alternativa, uma nova tecnologia ou uma nova ética, no lugar da que foi cooptada por empresas como a Microsoft e a Apple, o que seria representado pelo movimento Software Livre e pensadores como Lawrence Lessig. Do outro, existe uma recusa que simplesmente toma 
o que quer segundo uma ética própria que seria, nas palavras de Wark (2004, p. 20), "libertar os recursos produtivos e inventivos do mito da escassez". Essa vertente geralmente, mas não necessariamente, considera a imensa desigualdade da sociedade capitalista como intransponível, o que justificaria os meios empregados. Neste caso, referimo-nos à pirataria, mas também ao vazamento (leaks) e sabotagens (crackers/hackers como Anonymous). Vale ressaltar que, dentro da ideia de vínculo e modo de existência (SIMONDON, 2017), não é preciso ter consciência dessa condição. As ações já nascem formatadas por essa conjuntura que inclui desigualdade, precariedade e, principalmente, escassez (WARK, 2004).

Como Liang (2014) defende, existe uma pirataria transformadora. Ela está presente mais nos meios, nas tecnologias e estratégias suscitadas do que no conteúdo que é efetivamente "copiado". O mero ato de "copiar" pode se ramificar em diferentes formas de publicar, distribuir, baixar, procurar, considerar extensão e tamanho de entrada e saída de arquivos e, claro, os locais específicos onde fazê-lo. Por isso, não se trata de "manchar" a cultura hacker, "forçando" o uso do pirata como um de seus epítetos, mas, como defendem Castells (2003) e Wark (2004), a cultura hacker comporta todos esses significados. Por esse e outros motivos, certa intercambialidade entre hacker e pirata seria o ideal.

Segundo Alexander Galloway, por exemplo, a potencialidade do protocolo cria "classes" como a do hacker. O autor afirma que "a propriedade comercial do software é o principal empecilho detestado por todos os hackers porque significa que o código está limitado - limitado pelas leis de propriedades intelectual, limitados para fins de lucro, limitado pelos 'estraga-prazeres' das corporações"14 (2004, p. 170, tradução nossa), cabendo ao hacker responder a essa espécie de "chamado" e libertar o código dessa e de outras amarras. O hacker se sente compelido a remover todos os empecilhos ou ineficiências que podem atrapalhar esse desenvolvimento. Para o autor, hackers são cooptados pelo processo de crescimento ou "apoteose" do próprio código (Cf. GALLOWAY, 2004).

A manipulação de uma imagem ou vídeo para qualquer fim, seja de entretenimento ou político, seria atravessada por esse ethos hacker contemporâneo. Assim como o download de um filme, criação de mod ou crackeamento de um software, passando ainda por iniciativas governamentais de transparência' ${ }^{15}$, acesso

\footnotetext{
14 Do original: Commercial ownership of software is the primary impediment hated by all hackers because it means that code is limited-limited by intellectual property laws, limited by the profit motive, limited by corporate 'lamers'.

15 Dentro das ações da chamada e-democracia, definida como a busca por participação e transparência dentro da esfera governamental, a Câmara dos Deputados brasileira criou um Laboratório Hacker. Ver CÂMARA DOS
} 
e fomento da democracia ${ }^{16}$, entre outros. Diferente de uma cultura hacker, que acaba remetendo a uma ideia de pertencimento ou um local de fala, o modo de existência dos games e dos hackers replica a lógica do contágio, como o devir de Deleuze e Guattari (2008). Não é algo voluntário, mas produzido pelos vínculos.

Pensando na complementariedade entre os termos, Philip (2014) vê o pirata como um modo de tradução. Numa inspiração antropológica, através da figura do pirata (ou sua etnografia), enxergam-se modos de vida contemporâneos e outros pontos de vista para instâncias já estabelecidas. Neste caso: entretenimento, noções de letramento, criatividade, sociabilidades (formação de grupos e comunidades), política e mídia. Ela explica:

Objetos limítrofes não brilham primeiramente por si mesmos, mas como meios de tradução. Ver os piratas como objetos limítrofes ajuda a trazer para o foco os campos que se estabilizam em cada um de seus lados e assim ver como coemergentes a figura do pirata e os campos com os quais ela está sempre imbricada"7 (PHILIP, 2014, p. 165, tradução nossa).

As condições de existência do pirata, que a partir da presente argumentação podem ser vistas como as dos hackers, revelam formas de acessibilidade e recuperação da esfera comum, do domínio público (mesmo que à força, de forma informal ou marginal) na contemporaneidade. Em nossa perspectiva, piratas e hackers tornam-se figuras de mediação. Daí a necessidade de estudá-los em meios aos games, não como uma ruptura ou quebra, mas como a medida, ou melhor, um indicador de suas conexões - e, principalmente, como parte integrante da experiência de jogo vivenciada pelos hackers.

\section{Hackerismo como parte da experiência de jogo}

DEPUTADOS. Laboratório Hacker. 2013. Disponível em: http://www2.camara.leg.br/a-camara/estruturaadm/ diretorias/diretoria-geral/estrutura/copy_of_laboratorio-hacker. Acesso em: 1 jul. 2015.

16 Iniciado na Europa, 2006, há também um Partido Pirata do Brasil, fundado em 2012. Os Partidos Piratas da Suécia (o primeiro), República Tcheca, Alemanha e Islândia vêm inclusive concorrendo e vencendo eleições regionais, nacionais e transnacionais (europeias). BRASIL, Partido Pirata do. Home page do Partido Pirata. 2012. Disponível em: http://partidopirata.org/. Acesso em: 2 jul. 2012.

17 Do original: Boundary objects shine not primarily as themselves, but as a means of translation. Seeing pirates as boundary objects helps bring into focus the fields stabilizing on either side of the pirate, and thus to see as co-emergent the pirate figure along with the fields with which it is always imbricated. 
A definição de experiência de jogo perpassa muitas questões epistemológicas, desde os chamados estudos clássicos do lúdico - afinal, como estipular significados para uma ação que envolve diferentes atividades sempre ditas como tão envolventes, arrebatadoras, como o ato de jogar com o auxílio de computadores, a brincadeira infantil "tradicional", os esportes, além de outros tipos de competições? Para tanto, devemos voltar a esses estudos clássicos, especialmente a partir das inferências do historiador holandês Johan Huizinga, considerado o precursor dos estudos do lúdico no século $\mathrm{XX}$ :

O jogo é uma atividade ou ocupação voluntária, exercida dentro de
certos e determinados limites de tempo e espaço, segundo regras
livremente consentidas, mas absolutamente obrigatórias, dotado de
um fim em si mesmo, acompanhado de um sentimento de tensão e
de alegria e de uma consciência de ser diferente da "vida quotidiana"
(HUIZINGA, 2004, p. 33).

Ou seja, podemos inferir, a partir da declaração de Huizinga, que a atividade lúdica pode gerar uma sensação de separação do tempo-espaço do nosso dia a dia, regido por uma ordem diferente, que deve ser necessariamente aceita pelos jogadores para que eles sejam envolvidos pela experiência que eles vão construir a partir de sua ação no jogo. Ao pensarmos nesse "[...] espaço que o jogo se processa e que suas regras têm validade" (HUIZINGA, 2004, p. 23), percebemos que a atividade lúdica sempre deve estar em ação, nunca em potência, já que o jogo só existe quando operamos nele, a partir da aceitação das suas regras, e da atuação sobre elas. A experiência lúdica, então, pressupõe duas questões: 1) que o jogo é movimento, nunca se apresenta em latência; 2) que este jogo depende necessariamente de uma série de informações que se apresentam de forma externa à sua ação, como suas regras. Essas informações externas seriam o que Gregory Bateson chamou de "um certo grau de metacomunicação" (some degree of metacommunication), ou seja, a "[...] troca de signos que poderiam transportar a mensagem 'Isto é um jogo'”18 (BATESON, 2006, p. 316, tradução nossa). Esta afirmação de que o jogo envolve elementos externos ao seu ato em si, pode ser também encontrada em outros autores clássicos do lúdico, como o antropólogo Roger Caillois, que declara, logo no início do seu livro Les jeux et les hommes (Os jogos e os seres humanos):

18 Do original: [...] exchanging signals which would carry the message 'This is play'. 
[...] a palavra jogo não apenas denomina a atividade específica a qual nomeia, mas também a totalidade das figuras, dos símbolos ou dos instrumentos necessários a essa atividade, ou ao funcionamento de um conjunto complexo'9 (CAILLOIS, 1994, p. 9, tradução nossa).

Assim, podemos inferir que o jogo é uma ação em um mundo "[...] de totalidade fechada, completa em seu princípio e imutável..."20 (CAILLOIS, 1994, p. 9, tradução nossa), mas para a ação lúdica ocorrer, precisamos de vários outros elementos externos que possibilitam essa prática. Estes elementos podem ser físicos, como algoritmos e interfaces gráficas (no caso dos games), equipamentos e vestimentas específicas (nos esportes), ou brinquedos, quanto mesmo imateriais; como um elemento da cultura (HUIZINGA, 2004; CALLOIS, 1994), os jogos são naturalmente influenciados pelas culturas e sociedades humanas, assim como podem influenciá-los. Sobre esse aspecto da atividade lúdica, Bateson define que

[...] o jogo é um fenômeno no qual as ações de "jogo" estão relacionadas a, ou denotam, outras ações de "não-jogo". E então, nós nos encontramos no jogo a partir de uma instância de signos que fazem referência a outros eventos.... ${ }^{21}$ (BATESON, 2006, p. 317, tradução nossa).

Esta percepção de que existem subsídios da atividade lúdica que são externos ao jogo em si pode também ser encontrada entre os pesquisadores dos chamados game studies, que aplicam as teorias clássicas do lúdico ao estudo dos jogos eletrônicos. Por exemplo, em seu compêndio An Introduction to Game Studies (2008), Frans Mäyrä separa, didaticamente, o estudo de games em 1) o estudo dos jogos em si; 2) o estudo dos jogadores; 3) o estudo dos contextos que envolvem a interação entre jogos e jogadores, sempre demonstrando a importância dos elementos culturais deste tipo de ação, afirmando que

jogar é, fundamentalmente, uma forma de contato a partir da interação, e durante o ato de jogar, a coisa mais importante é manter essa interação rolando, e desenvolver os seus resultados, previsíveis

\footnotetext{
19 Do original: [...] la palabra juego designa no solo la actividad específica que nombra, sino también la totalidad de las figuras, de los símbolos o de los instrumentos necesarios a esa actividad o al funcionamiento de un conjunto complejo.

20 Do original: [...] de totalidad cerrada, completa en un principio e inmutable...".

${ }^{21}$ Do original: [...] play is a phenomenon in which the actions of 'play' are related to, or denote, other actions of 'not play'. We therefore meet in play with an instance of signals standing for other events...
} 
ou surpreendentes. O conceito de cultura dos games pode ajudar a destacar o pano de fundo, quase não mencionado, no qual os jogos fazem sentido para seus jogadores ${ }^{22}$ (MÄYR $\ddot{A}, 2008$, p. 14, tradução nossa).

Ao estudar fundamentos para uma epistemologia dos games, Espen Aarseth (2003, p. 2) demonstra três "dimensões" que caracterizariam todos os jogos presentes em ambientes virtuais: o gameplay (as ações dos jogadores, suas estratégias e motivações), a estrutura dos jogos em si (suas regras gerais de funcionamento, tanto das ações quanto do ambiente), e o "mundo" do game (seu conteúdo ficcional, o design de suas fases). Cada uma dessas dimensões pode ser pesquisada a partir dos seus campos constituintes:

[...] focando em cada um destes três níveis, podemos identificar três tipos diferentes de perspectivas para a pesquisa de games:

- Gameplay: sociológica, etnológica, psicológica etc.

- Regras do jogo: Game Design, mercado, leis, Computação/Inteligência Artificial. ${ }^{23}$ (ARSETH, 2003, p. 3, tradução nossa).

Já Katie Salen e Eric Zimmerman (2004, p. 4), pesquisadores ligados à área do design de games, ou seja, mais interessados em entender como os jogos eletrônicos se desenvolvem como uma linguagem particular, preferem trabalhar com um "esquema primário" (primary schema) dos games, que envolve regras (rules), o ato de jogar (play) e a cultura (culture).

Estas definição tripartidas de Mäyrä (2008), Aarseth (2003), Salen e Zimmerman (2003) sobre os games parecem ser inteiramente baseadas nos chamados estudos clássicos do lúdico, se seguirmos a sugestão de Jesper Juul (2005, p. 197) de que existem três níveis de descrição sobre jogos feitos por estas correntes: o jogo em si, a relação do jogador com o jogo e a relação entre o ato de jogar e o resto do mundo. Neste sentido, Juul constrói sua teoria do

22 Do original: playing is fundamentally a form of contact by interaction, and while playing, it is most important that we keep the interplay going, and derive its predictable or surprising outcomes. A concept of games culture can help to bring into light the mostly unspoken backdrop against which games make sense for their players.

23 Do original: by focusing on each of the three levels, we could identify three different types of games research perspectives: / Gameplay: sociological, ethnological, psychological etc. / - Game-rules: Game Design, business, law, computer science/AI / • Game-world: Art, aesthetics, history, cultural/media studies, economics. 
lúdico digital buscando conscientemente as raízes das pesquisas sobre o lúdico, definindo sua abordagem como sendo uma relação entre esses níveis "clássicos" com uma interseção entre os games como regras e o game como um produto ficcional (JUUL, 2005, p. 197), o que também está presente nas abordagens que citamos acima, embora com outras formas didáticas de enfoque. Ou seja, neste tripé das epistemologias do lúdico, tanto nos formatos ditos tradicionais ou nas implementações eletrônicas, podemos sempre observar a presença da experiência de jogo, referenciada principalmente a partir dos estudos do envolvimento do jogador com o universo construído pelo jogo, seja a partir de suas ações, no momento da interação com as regras e outros elementos físicos (que permitem ao jogo sair de sua latência e entrar em movimento), seja a partir dos elementos culturais que envolvem essa interação entre o jogador e sua ação lúdica.

Para os objetivos deste artigo, é interessante observar que a maioria dos autores voltados ao estudo dos games se referem à experiência de jogo como sendo apenas a relação entre o jogador em relação à mecânica do jogo - a experiência que é definida pelo game designer durante o seu processo de criação, apresentada ao jogador a partir de sua interação com os hardwares e softwares que materializam o mundo do jogo. Entendemos que a própria materialidade dos jogos eletrônicos evidencia o contorno deste tipo de pensamento, pois visto de forma meramente técnica, o game nada mais é do que uma sequência qualquer de algoritmos, executada por uma máquina digital. Neste sentido, o algoritmo do jogo é apenas uma das condições materiais para que esta experiência de jogo ocorra, da mesma forma que um campo de futebol precisa ser preparado (com aparagem da grama, demarcação de limites, colocação de traves e redes no gol etc.) para uma partida profissional poder ter seu início. Contudo, assim como uma partida de futebol precisa da bola rolando após o apito do juiz para ser considerada como tal, o game só existe como atividade lúdica a partir do processo de comunicação entre o jogador e a máquina, a interação humano-computador necessária para a operação de qualquer dispositivo digital. Ou seja, o jogo eletrônico só existe como atividade lúdica a partir dessa conexão, quando deixa de estar em potência no universo construído em seu código operacional e entra em movimento, gerando a experiência de jogo tão necessária para a imersão do jogador.

Pensar, porém, apenas na experiência de jogo como a ação de contato entre humanos e máquinas não nos parece suficiente para definir esse processo, já que este enfoque pode ocultar outros elementos necessários ao processo lúdico, como a produção de sentido durante/a partir destas atividades; para o peda- 
gogo francês Gilles Brougère, "o jogo é o resultado de relações interindividuais, portanto de cultura" (1998, p. 189), acrescentando também que "o jogo não é um comportamento específico, mas uma situação na qual esse comportamento adquire uma significação específica. Vê-se que o jogo supõe comunicação e interpretação" (1998, p. 191). É justamente devido a essa produção de sentidos que ocorre dentro do universo lúdico que Huizinga afirma ser o lúdico “[...] uma função significante, isto é, encerra um determinado sentido [...]. Todo jogo significa alguma coisa" (2004, p. 3-4). Essa noção não pertence somente aos estudos clássicos do lúdico, sendo também apresentada pelos estudos de jogos eletrônicos; Frans Mäyrä divide a estrutura do jogo em um "núcleo" (core) e sua "casca" (shell), ou seja, em mecânicas de jogo (regras e modos de interação jogador/jogo) e as representações/signos que constituem o jogo, já que:

Os sentidos nos games são criados a partir de interações lúdicas que acontecem dentro de contextos culturais específicos. Estes sentidos estão relacionados tanto aos jogos, que que transmitem significados na forma de comunicação simbólica, quanto aos significados não-simbólicos que são inerentes ao ato de brincar e ao gameplay total24 (MÄYRÄ, 2008, p. 27-28, tradução nossa).

É justamente essa produção imaterial de sentidos que corresponde ao não-jogo citado por Bateson como essencial à atividade lúdica, sendo parte integral não só dos games, mas igualmente da lida com os dispositivos digitais em geral, por meio da interação humano-computador. Em The Computer as a Communication Device, um dos primeiros escritos que refletiram sobre as questões inerentes aos processos de comunicação mediados por computador, J.C.R. Licklider e Robert W. Taylor descrevem que os usuários computacionais agem “[...] como participantes ativos em um processo contínuo, trazendo algo para este processo a partir das nossas interações com ele, e não somente recebendo algo dele, pela nossa conexão com ele" (LICKLIDER; TAYLOR, 1990, p. 21, tradução nossa), afirmando também que,

Comunicar-se é mais do que enviar e receber [informações]. Por acaso, dois gravadores de fita se comunicam quando eles tocam um para o outro, e gravam a partir do outro? Não mesmo - não no nosso

24 Do original: Meanings in games are created in playful interactions that take place within specific cultural contexts. Such meanings are related both to games conveying meaning in the manner of symbolic communication, and to the non-symbolic meanings inherent in the act of playing and in the overall gameplay experience. 
sentido [humano]. Nós acreditamos que os comunicadores devem fazer algo não-trivial com a informação que eles enviam e recebem ${ }^{25}$ (1990, p. 21, tradução nossa).

Reflexões como estas de Licklider e Taylor (1990) ajudaram a construir a subárea das Ciências da Computação que lida com os processos de comunicação entre usuários e suas máquinas: a experiência do usuário (também conhecida pela sigla UX, derivada do inglês User Experience). Esse termo, popularizado por Donald Norman a partir da metade dos anos 1990, foi diretamente inspirado pelas observações feitas pelos primeiros pesquisadores da computação em relação aos jogos eletrônicos, por exemplo, no clássico artigo Heuristics for Designing Enjoyable User Interfaces: Lessons from Computer Games, publicado em 1982, por Thomas W. Malone, que propunha o uso de características lúdicas (como o desafio, a ficção e a curiosidade) para o design de ambientes digitais. O conceito de manipulação direta (direct manipulation), descrita como a sensação de manipular os dados computacionais por meio de representações visuais, agindo de forma ativa em um ambiente de interface, também foi inspirado nas experiências de jogo advindas dos games; em Direct Manipulation: a Step Beyond Programming Languages (1983), texto que popularizou esta expressão, Ben Shneiderman deixa bem clara a sua inspiração:

Provavelmente, o emprego mais excitante, mais engenhoso - e certamente mais bem-sucedido - da manipulação direta está no mundo dos videogames [...] Os designers desses games nos forneceram uma forma de entretenimento estimulante, um desafio para novatos e experts, e muitas lições intrigantes sobre os fatores humanos do design de interfaces [...] A forte atração trazida por esses jogos contrastam marcadamente com o nervosismo e a resistência que muitos usuários vivenciam no uso de equipamentos automatizados no trabalho' ${ }^{26}$ (SHNEIDERMAN, 1983, p. 61, tradução nossa).

25 Do original: But to communicate is more than to send and to receive. Do two tape recorders communicate when they play to each other and record from each other? Not really - not in our sense. We believe that communicators have to do something nontrivial with the information they send and receive.

26 Do original: "Perhaps the most exciting, well-engineered - certainly, the most successful - application of direct manipulation is in the world of video games [...]The designers of these games have provided stimulating entertainment, a challenge for novices and experts, and many intriguing lessons in the human factors of interface design [...] The strong attraction of these games contrasts markedly with the anxiety and resistance many users experience toward office automation equipment. 
Nesse sentido, notamos que o próprio conceito de interação humano-computador foi diretamente influenciado pela ontologia das atividades lúdicas, procurando reproduzir o elemento essencial da experiência de jogo. Porém, retomando as discussões anteriores, entendemos que a experiência de jogo não está somente relacionada com essa interação entre humanos e máquinas, já que: 1) a interação não é realizada apenas com o jogo em si, mas também com suas regras e elementos físicos constituintes, como seus algoritmos e interfaces físicas; 2) o próprio conceito de experiência enfatiza seu aspecto social, pois envolve trocas entre pessoas, que acontecem a partir de contatos mútuos - um exemplo está na forma em que John Dewey descreve a experiência gerada pelos processos educacionais:

Uma experiência é sempre o que ela é por conta de uma transação que acontece entre um indivíduo e o que constitui o seu ambiente, naquele momento, seja o ambiente constituído de pessoas com as quais ele está falando sobre algum tópico ou evento, o assunto sobre o qual se fala também sendo parte da situação; ou sobre os brinquedos com os quais ele está brincando; o livro que ele está lendo (no qual suas condições ambientais naquele momento podem ser a Inglaterra, ou a Grécia Antiga, ou uma região imaginária; ou os materiais de um experimento que ele está realizando. $O$ ambiente, em outras palavras, são quaisquer condições que interagem com necessidades especiais, desejos, motivações e as capacidades para criar a experiência que está acontecendo. Mesmo quando a pessoa constrói castelos ao vento, ele está interagindo com os objetos que ele constrói na sua imaginação ${ }^{27}$ (DEWEY, 1997, p.43-44, tradução nossa).

Assim, entendemos que a experiência de jogo não está restrita apenas à jogabilidade, ou seja, ao contato do jogador com o universo ficcional do game, que é moldada pelos seus designers, mas a todos os seus aspectos constituintes, o que envolve também os aspectos sociais do ato de jogar - compartilhar as experiências com amigos ou com uma comunidade de entusiastas, por exem-

27 Do original: An experience is always what it is because of a transaction taking place between an individual and what, at the time, constitutes his environment, whether the latter consists of persons with whom he is talking about some topic or event, the subject talked about being also a part of the situation; or the toys with which he is playing; the book he is reading (in which his environing conditions at the time may be England or ancient Greece or an imaginary region); or the materials of an experiment he is performing. The environment, in other words, is whatever conditions interact with personal needs, desires, purposes, and capacities to create the experience which is had. Even when a person builds a castle in the air he is interacting with the objects which he constructs in fancy. 
plo - mas também em interagir com a sua própria materialidade constituinte, o que implica, no caso dos games, em realizar modificações no seu código-fonte, hackear sua estrutura.

Em verdade, hackerismo e os games estão intrinsicamente ligados, pois, como nos explica Pekka Himanen:

[...] o hacker programa porque acha a programação uma atividade intrinsecamente interessante, excitante e lúdica [...] O princípio geral é esse: os hackers programam porque os desafios da programação são interessantes. Os problemas encontrados na programação causam curiosidade nos hackers e os tornam ávidos por mais conhecimento (HIMANEN, 2001, p. 19).

Esta busca pelo conhecimento e pela diversão torna-se ainda mais evidente ao retornarmos ao início do desenvolvimento de softwares (especialmente games), no começo dos anos 1970, quando os computadores começaram a deixar os laboratórios de pesquisa para se disponibilizarem ao público de hobbyistas eletrônicos. No início, estes entusiastas estavam mais interessados na montagem de suas máquinas, na elaboração do hardware computacional, porém, a partir do momento que o mercado de microcomputadores se ampliou, passando a oferecer máquinas prontas de baixo custo (Cf. WATSON, 2012; CERUZZI, 2012) que precisavam apenas do uso de programação para o seu funcionamento, os softwares tomaram a primazia dos hobbyistas. Neste período, as revistas voltadas para a computação passaram a oferecer exemplos de softwares para uso pessoal, com o oferecimento de códigos em diferentes linguagens para a reprodução dos programas criados pelos hobbyistas, como a brasileira Micro Sistemas, que veiculou alguns dos primeiros jogos desenvolvidos por designers nacionais.

\section{Considerações finais}

A cultura dos hackers sempre se valeu da livre distribuição e modificação de conteúdos digitais, e isso era válido também para os games, que nesta época correspondiam a aproximadamente $60 \%$ do mercado de softwares no começo dos anos 1980 (Cf. CAMPBELL-KELLY, 2003, p. 276). Contudo, a partir desta exploração comercial, que teve seu início no final dos anos 1970, mexer no código-fonte passou a ser desencorajado, até mesmo proibido, ganhando conotação negativa. Um marco desta transformação de pensamento foi o texto An Open Letter to Hobbyists, escrito por Bill Gates em fevereiro de 1976, e divulgada na 
newsletter do Homebrew Computer Club da região estadunidense do Vale do Silício. Nesta carta, Gates afirma seu descontentamento com hobbyistas que copiavam softwares da Microsoft:

\begin{abstract}
Uma coisa que vocês fazem é prevenir que bons softwares sejam escritos. Quem pode trabalhar profissionalmente de graça e se manter? Qual hobbyista pode gastar três anos da sua vida programando, descobrindo todos os bugs, fazer a documentação do seu produto, e distribuí-lo de graça? [...] o que vocês fazem é roubo ${ }^{28}$ (GATES, 1976, p. 2, tradução nossa).
\end{abstract}

Nesse sentido, a carta de Gates nos mostra também a mudança na conotação da palavra hackear, que passou a denominar atos de desrespeito às leis de direitos autorais, passando de uma parte realmente ativa da experiência de jogo, nesses primórdios, para algo proibido. O hackerismo, parte essencial da experiência dos jogos eletrônicos desde o seu início, foi desencorajado dessa transição, já que o domínio do código passa a ser regido por regras de copyright. Assim, mais do que um movimento de resistência político-ideológica, os mods nos mostram o DNA, traçam a origem dos games, ao reforçar a criação como parte da (meta) experiência de jogo - revelando o hackerismo como ato de exploração ativo, uma atividade essencialmente lúdica. Tentar entender os conceitos relacionados ao hackerismo nos games acaba por ampliar o ato do jogo digital para ações que estariam "fora" do ambiente do jogo em si (a apenas interação jogo/jogador dentro dos limites pré-determinados pelos seus designers). Hackear um game, ou seja, modificar, customizar, interferir na sua materialidade constituinte (seu código-fonte), é uma ação de natureza sociocultural, portanto, que estaria inclusa dentro da experiência de jogo, para aqueles que assim desejarem.

\title{
Referências
}

ARSETH, Espen. Playing Research: Methodological approaches to game analysis. In: SPILFORSKNING.DK CONFERENCE, 2003, Denmark. Proceedings [...]. Denmark, 2003.

AMARAL, Adriana. MONTEIRO, Camila. "Esses roqueiro não curte": performance de gosto e fãs de música no Unidos Contra o Rock do Facebook. In: Revista Famecos, v. 20, n. 2, maio/ago. 2013. p. 446-471. https://doi.org/10.15448/1980-3729.2013.2.15130

28 Do original: One thing you do do is prevent good software from being written. Who can afford to do professional work for nothing? What hobbyist can put 3-man years into programming, finding all bugs, documenting his product and distribute for free? (...) the thing you do is theft. 
BATESON, Gregory. A theory of play and ambiguity. In: SALEN, Katie; ZIMMERMAN, Eric (ed.). The game design reader: a rules of play anthology. Cambridge: MIT Press, 2006. BROUGÈRE, Gilles. Jogo e educação. Porto Alegre: Artmed, 1999.

CAILLOIS, Roger. Los juegos y los hombres: la máscara y el vértigo. México, D.F.: Fondo de Cultura Económica, 1994.

CAMPBELL-KELLY, Martin. From airline reservations to Sonic the Hedgehog: a history of the software industry. Cambridge: MIT Press, 2003.

CASTELLS, Manuel. A Galáxia Da Internet: reflexões sobre a internet, os negócios e a sociedade. Rio de Janeiro, Zahar, 2003.

CERUZZI, Paul. Computing: a concise history. Cambridge: MIT Press, 2012. https://doi. org/10.7551/mitpress/9426.001.0001

CONSALVO, Mia. There is No Magic Circle. Games and Culture, v. 4, n. 4, p. 408-417, 2009. https://doi.org/10.1177/1555412009343575

DELEUZE, Giles. Nietzsche e a filosofia. São Paulo: N-1 edições, 2018.

DEWEY, John. Experience and Education. New York: Touchstone, 1997.

GALLOWAY, Alexander R. Protocol: how control exists after decentralization. Cambridge: MIT Press, 2004. https://doi.org/10.7551/mitpress/5658.001.0001

GALLOWAY, Alexander R. Gaming: Essays on Algorithmic Culture. Minneapolis: University of Minnesota Press, 2006.

GATES, Bill. An open letter to hobbyists. Homebrew Computer Club Newsletter, v. 1, n. 2, p. 2, 1976.

GEE, James Paul. Literacy And Education. New York: Routledge, 2015. https://doi. org/10.4324/9781315739571

GEE, James Paul; HAYES, Elisabeth R. Women and Gaming: The Sims and 21st Century Learning. New York: Palgrave Macmillan, 2010. https://doi.org/10.1057/9780230106734

HENNION, Antoine. Loving Music: from a Sociology of Mediation to a Pragmatics of Taste. Comunicar, v. 17, n. 34, p. 25-33, 2010. https://doi.org/10.3916/C34-2010-02-02

HIMANEN, Pekka. The Hacker Ethic: a radical approach to the philosophy of business. New York: Random House, 2001.

HUIZINGA, Johan. Homo ludens. 5. ed. São Paulo: Perspectiva, 2004.

JUUL, Jesper. Half-real: Video games between real rules and fictional worlds. Cambridge: MIT Press, 2005. 
LATOUR, Bruno. Reassembling the social: an introduction to Actor-Network-Theory. New York: Oxford University Press, 2005.

LATOUR, Bruno. An Inquiry into Modes of Existence: An Anthropology of the moderns. Cambridge, Massachusetts: Harvard University Press, 2013.

LIANG, Lawrence. Beyond Representation: The Figure of the Pirate. In: ECKSTEIN, Lars; SCHWARZ, Anja (Ed.). Postcolonial Piracy: Media Distribution and Cultural Production in the Global South. New York: Bloosmbury, 2014.p. 49-78

LICKLIDER, J.C.R.; TAYLOR, Robert W. The Computer as a Communication Device. In: SRC - Digital Systems Research Center. In Memoriam: J. C. R. Licklider. Palo Alto: SRC, 1990. p. 21-47. Technical Report.

MALABY, Thomas M. Beyond Play: A New Approach to Games. Games and Culture, v. 2, n. 2, p. 95-113, 2007. https://doi.org/10.1177/1555412007299434

MALONE, Thomas W. Heuristics for designing enjoyable user interfaces: lessons from computer games. In: CONFERENCE ON HUMAN FACTORS IN COMPUTER SYSTEMS, 1982, Gaithersburg: Proceedings [...]. Gaithersburg: Association for Computing Machinery - ACM, 1982. https://doi.org/10.1145/800049.801756

MÄYRÄ, Franz. An Introduction to Game Studies: games in culture. London: SAGE, 2008.

MATEAS, Michael. Procedural Literacy: Educating the New Media Practitioner. In: DAVIDSON, Drew (org.). Beyond Fun: Serious Games and Media. [S. I.]: ETC, 2008

MOULTHROUP, S.. "From work to play: molecular culture in the time of deadly games". In: Wardrip-Fruin, N. \& Harrigan, P. (Org.). First Person: new media as story, performance and game. Cambridge: MIT Press, 2004.

NATALE, Adriano. A ciência dos videogames: tudo dominado....pelos elétrons. Rio de Janeiro: Vieira \& Lent, 2013.

NEGRI, Antonio; HARDT, Michael. Multidão. Rio de Janeiro: Record, 2005.

NEGRI, Antonio; HARDT, Michael. Império. 9. ed. Rio de Janeiro: Record, 2010.

PHILIP, Kavita. Keep on Copyin' in the Free World? Genealogies of the Postcolonial Pirate Figure. In: ECKSTEIN, Lars; SCHWARZ, Anja. Postcolonial Piracy: Media Distribution and Cultural Production in the Global South. New York: Bloosmbury, 2014.

REGIS, Fátima. Textos, texturas e intertextos: apontamentos sobre aprendizado e competência na comunicação digital. In: ENCONTRO ANUAL DA COMPÓS, 23, 2014. Anais [...]. Belém: UFPA, 2014.

SHNEIDERMAN, Ben. Direct manipulation: a step beyond programming languages. IEEE Computer, v. 16, n. 8, 1983. https://doi.org/10.1109/MC.1983.1654471 
SIMONDON, Gilbert. On the Mode of Existence of Technical Objects. Minneapolis: Univocal, 2017.

TIMPONI, Raquel; REGIS, Fátima. Legere et interpretari: letramentos midiáticos como metodologia de aprendizagem. Reciis, v. 4, n. 11, p. 1-11, out. /dez. 2017. https://doi. org/10.29397/reciis.v11i4.1119

WARK, McKenzie. A Hacker Manifesto. Cambridge: Harvard University Press, 2004.

WATSON, Ian. The Universal Machine: From the Dawn of Computing to Digital Consciousness. Berlin: Springer-Verlag, 2012. https://doi.org/10.1007/978-3-642-28102-0

ZIMMERMAN, Eric. Gaming Literacy: Game Design as a Model for Literacy in the Twenty-First Century. In: PERRON, Bernard; WOLF, Mark J. P. Video Game Theory Reader 2. New York: Routledge, 2009.

\section{Dados dos autores:}

José Messias - jmessias.santos@gmail.com

Bolsista de Pós-Doutorado (Capes/PNPD) no Programa de Pós-Graduação em Comunicação da Universidade Federal Fluminense - UFF. Doutor em Comunicação e Cultura pela Escola de Comunicação da Universidade Federal do Rio de Janeiro - UFRJ.

Endereço do autor: Universidade Federal Fluminense, Instituto de Arte e Comunicação Social. Rua Professor Marcos Waldemar de Freitas Reis, s/n, Bloco A, $4^{\circ}$ andar, São Domingos 24.210-201 - Niterói (RJ) - Brasil

\section{Letícia Perani - leticiaperani@yahoo.com.br}

Professora adjunta do Instituto de Artes e Design da Universidade Federal de Juiz de Fora - UFJF. Doutora em Comunicação pelo PPGCom da Universidade Estadual do Rio de Janeiro - UERJ.

Endereço da autora: Universidade Federal de Juiz de Fora, Instituto de Artes e Design. Campus Universitário, s/n, São Pedro, 36.036-900 - Juiz de Fora (MG) - Brasil 